\title{
Improved theory of forced magnetic reconnection due to boundary perturbation
}

\author{
A. Ishizawa* and S. Tokuda \\ Department of Fusion Plasma Research, Naka Fusion Research Establishment, \\ Japan Atomic Energy Research Institute, Naka, Ibaraki 311-0193, Japan
}

(Received May 30, 2000; Revised November 27, 2000; Accepted February 1, 2001)

\begin{abstract}
Boundary layer theory of forced magnetic reconnection due to an externally applied boundary perturbation is improved by introducing correct asymptotic matching which takes into account the effect of inertia in the inner layer precisely. The improved theory yields an integral equation for the time evolution of the reconnected flux. The initial evolution of the reconnection process is characterized by some significant features represented by the reconnected flux and an inner-layer reconnected flux. The typical time scales of the reconnected flux and of the inner-layer reconnected flux include the time scale of the boundary perturbation and exclude the Sweet-Parker time scale. The role of stability against the tearing modes in the reconnection process is also clarified. A current sheet induced on a resonant surface is in such a direction as to oppose the progress of the reconnection, because the equilibrium is stable against tearing modes in the absence of the boundary perturbation.
\end{abstract}

\section{Introduction}

There are two kinds of magnetic reconnections: free reconnection and forced reconnection. Free reconnection is caused by spontaneous instabilities such as the tearing mode (Furth et al., 1963). Even if a magnetic equilibrium is stable for resistive modes, an externally imposed boundary perturbation gives rise to magnetic reconnection called forced reconnection (Hahm and Kulsrud, 1985; Wang and Bhattacharjee, 1992; Ma et al., 1996; Ishizawa and Tokuda, 2000). The forced reconnection is of interest in laboratory plasmas such as the island formation by resonant error fields (Fitzpatrick and Hender, 1991; Wang and Bhattacharjee, 1997) and the seed island formation for the neo-classical tearing mode (Hegna et al., 1999) and astrophysical plasmas such as the solar corona and the earth's magnetotail (Vekstein and Jain, 1998, 1999; Wang et al., 1998).

In order to understand the process of forced reconnection, we consider a deformation of the plasma boundary (Fig. 1) and follow the subsequent time evolution of the plasma. There are two equilibria which are consistent with this deformation: equilibrium (I) and equilibrium (II) (Hahm and Kulsrud, 1985), (Fig. 2). The equilibrium (I) has the same topology as the original equilibrium, while it has a current sheet on the resonant surface. The equilibrium (II) has the different topology with magnetic islands on the resonant surface and no current sheet. Equilibria intermediate between these two are also possible and consistent with the boundary deformation and represent the subsequent time evolution of the plasma. The time evolution of the intermediate equilibrium is determined by a time dependent coeffi-

*Present address: Graduate School of Energy Science, Kyoto University, Uji, Kyoto 611-0011, Japan.

Copy right (c) The Society of Geomagnetism and Earth, Planetary and Space Science (SGEPSS); The Seismological Society of Japan; The Volcanological Society of Japan; The Geodetic Society of Japan; The Japanese Society for Planetary Sciences. cient, called the reconnected flux. The time development of the reconnected flux is calculated by use of boundary layer theory as an initial value problem (Hahm and Kulsrud, 1985; Wang and Bhattacharjee, 1992; Ma et al., 1996). In the previous works, the effect of the inertia in the inner layer is neglected in the asymptotic matching. We introduce the appropriate asymptotic matching and adopt the exact solution for the inner layer equation to take into account precisely the effect of inertia in the inner layer (Ishizawa and Tokuda, 2000).

In this paper, we will present a new method to determine the time evolution of the reconnected flux which describes the improved reconnection process. The correct asymptotic matching yields the integral equation for the reconnected flux. This integral equation exhibits the new time evolution of the reconnected flux and of the current sheet induced on the resonant surface. Furthermore, the role of stability against the tearing modes in the reconnection process is clarified in terms of the tearing mode stability parameter.

\section{Boundary Layer Theory of Forced Reconnec- tion}

We consider the response of a slab of incompressible plasma to an applied boundary perturbation (Fig. 1). The plasma is supposed to be bounded by two parallel perfectly conducting walls. The magnetic field is represented by $\boldsymbol{B}=$ $B_{T} \boldsymbol{e}_{z}+\boldsymbol{e}_{z} \times \nabla \psi$, where $B_{T}$ stands for a uniform toroidal field and $\psi$ is a magnetic potential. We assume that the $x y$-plane is normal to the toroidal field, while the $y$-axis is parallel to the wall and the $x$-axis normal to it.

We adopt the reduced MHD equations,

$$
\begin{aligned}
\rho\left(\frac{\partial}{\partial t}+\boldsymbol{v} \cdot \nabla\right) \nabla^{2} \varphi & =\boldsymbol{B} \cdot \nabla j_{z}, \\
\frac{\partial \psi}{\partial t}+\boldsymbol{v} \cdot \nabla \psi & =\frac{\eta}{4 \pi} \nabla^{2} \psi,
\end{aligned}
$$




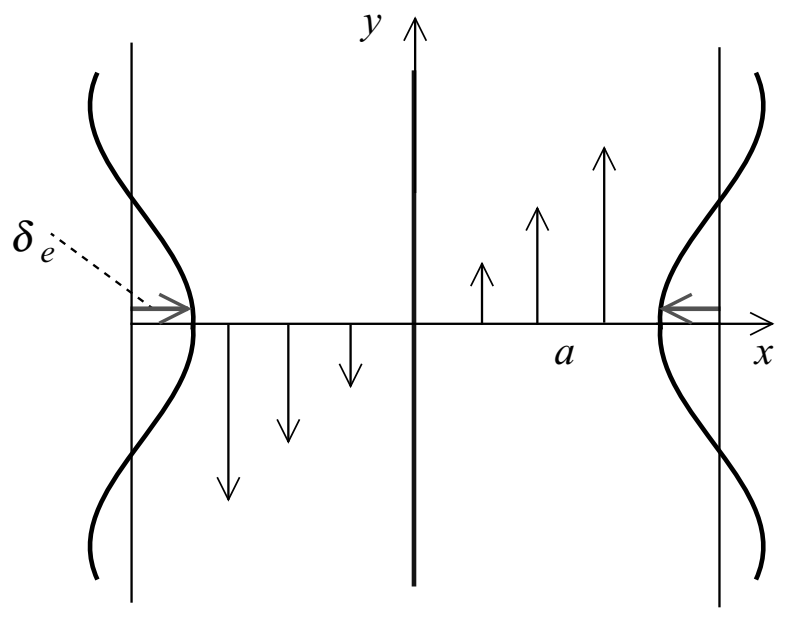

Fig. 1. Coordinate system for the slab of incompressible plasma.

where $j_{z}=\nabla^{2} \psi / 4 \pi$ and $\boldsymbol{v}=\boldsymbol{e}_{z} \times \nabla \varphi$ indicate the $z$ component of the current density and the velocity of the plasma respectively, and $\varphi$ is a stream function. The constants $\eta$ and $\rho$ denote the magnetic diffusivity and the density of the plasma.

\subsection{Outer region}

We assume that the outer region is quasi-static and is governed by the ideal MHD equation. Thus, the right-hand side of Eq. (2) can be zero as

$$
\boldsymbol{B} \cdot \nabla j_{z}=0 .
$$

Let us impose a boundary perturbation on the original static equilibrium, $\psi=\psi_{0}(x)$, subjected to the boundary conditions $\psi_{0}( \pm a)=$ const. This original equilibrium is assumed to have the resonant surface at the center of the plasma, $\psi_{0}^{\prime}(0)=0$, and is supposed to be stable for the usual tearing mode. The boundary perturbation is described in terms of a deformed plasma boundary as

$$
\psi(x= \pm(a-\delta \cos k y))=\text { const } .
$$

where $k$ and $\delta$ are the wave number and the amplitude of the boundary deformation, respectively. The boundary perturbation is assumed to be weak, $\delta \ll a$, such as the error fields in a tokamak. In this work, we consider a time varying boundary perturbation, $\delta=\delta\left(t / \tau_{e}\right)$. The time scale of the deformation $\tau_{e}$ is assumed to be much slower than the Alfvén time scale but much faster than the resistive time scale, $\tau_{A} \ll \tau_{e} \ll \tau_{R} ; \tau_{A}$ and $\tau_{R}$ are defined later.

The magnetic potential perturbed by the boundary deformation is written as

$$
\psi(x, t)=\psi_{0}(x)+\psi_{1}(x, t) \cos k y
$$

where $\psi_{1}(x, t)$ denotes the perturbed part due to the boundary perturbation. Since the perturbation is imposed on a time scale much slower than the Alfvén time scale, the plasma is quasi-static and obeys the ideal MHD equilibrium equation except in the vicinity of the resonant surface, where $x=0$. The ideal MHD equilibrium equation, Eq. (3), for the per- turbation $\psi_{1}(x, t)$ is reduced to

$$
\begin{gathered}
\psi_{0}^{\prime}(x)\left\{\frac{\partial^{2} \psi_{1}(x, t)}{\partial^{2} x}-k^{2} \psi_{1}(x, t)\right\} \\
-\psi_{0}^{\prime \prime \prime}(x) \psi_{1}(x, t)=0,
\end{gathered}
$$

with the boundary condition $\psi_{1}( \pm a, t)=\delta\left(t / \tau_{e}\right) \psi_{0}^{\prime}(a) \equiv$ $\psi_{e}\left(t / \tau_{e}\right)$. We consider a time dependent boundary perturbation as $\psi_{e}\left(t / \tau_{e}\right)=\psi_{e}^{\prime \prime}(0) t^{2} /\left(2 ! \tau_{e}^{2}\right)+\cdots$.

The solution to Eq. (5) is written as

$$
\psi_{1}(x, t)=\psi_{1}(0, t) f(x)+\psi_{e}\left(t / \tau_{e}\right) g(x),
$$

where $f(x)$ and $g(x)$ satisfy Eq. (5), and are subject to the boundary conditions $f(0)=1, f( \pm a)=0$ and $g(0)=0$, $g( \pm a)=1$. The first term is related to the tearing mode. Equilibrium (I) is described by $\psi_{1}(0, t)=0$ and $\psi_{e}(t) \neq 0$. The current sheet vanishes when $\psi_{1}(0, t) f^{\prime}(0)+$ $\psi_{e}(t) g^{\prime}(0)=0$, and thus it corresponds to equilibrium (II) (Fig. 3).

The quasi-static equilibrium, Eq. (6), is determined only by the reconnected flux $\psi_{1}(0, t)$, because $\psi_{e}\left(t / \tau_{e}\right)$ is assumed to be a given function.

We consider the time evolution of the quasi-static equilibrium as an initial-value problem, by applying the Laplace transform $\tilde{f}(x, s)=\int_{0}^{\infty} f(x, t) e^{-s t} d t$, to the outer-solution, Eq. (6). Then we derive the expansion to the outer solution as

$$
\begin{gathered}
\tilde{\psi}_{1}(x, s)=\tilde{\psi}_{1}(0, s)+\tilde{\psi}_{1}(0, s) \frac{\Delta_{\text {out }}^{\prime}}{2} x+\cdots, \\
x \rightarrow+0,
\end{gathered}
$$

where

$$
\begin{aligned}
\Delta_{\text {out }}^{\prime}(s) & \equiv \frac{1}{\tilde{\psi}_{1}(0, s)}\left[\frac{d \tilde{\psi}_{1}(x, s)}{d x}\right]_{-0}^{+0} \\
& =\Delta_{0}^{\prime}+\Delta_{s}^{\prime} \frac{\tilde{\psi}_{e}(s)}{\tilde{\psi}_{1}(0, s)},
\end{aligned}
$$

and where $\Delta_{0}^{\prime}=[d f(x) / d x]_{-0}^{+0}, \Delta_{s}^{\prime}=[d g(x) / d x]_{-0}^{+0}$. The first term, $\Delta_{0}^{\prime}$, is the tearing mode stability parameter in the absence of the boundary perturbation. Since the original static equilibrium is supposed to be stable, $\Delta_{0}^{\prime}$ is negative.

\subsection{Inner layer}

In order to obtain the reconnected flux we should investigate the dynamics in the vicinity of the resonant surface, i.e. the inner layer. In the analysis of the inner layer, it is important to include not only the resistivity but also the inertia of the plasma correctly.

We apply the Laplace transform to the linearized reduced MHD equations. The initial conditions for the perturbations are $\psi_{1}(x, 0)=\varphi_{1}(x, 0)=0$, because there is no deformation of the boundary at $t=0, \psi_{e}(0)=0$. By stretching the variables in the vicinity of the resonant surface according to $\hat{x}=x /(\varepsilon a), \hat{s}=\tau_{c} s, \varepsilon=\left(\tau_{A} / \tau_{R} k a\right)^{1 / 3}, \tau_{c}=\tau_{A} /(\varepsilon k a)$, the equations in the inner layer become

$$
\begin{aligned}
\hat{s} \frac{d^{2} \tilde{\varphi}_{i n}}{d \hat{x}^{2}} & =-\hat{x} \frac{d^{2} \tilde{\psi}_{i n}}{d \hat{x}^{2}}, \\
\hat{s} \tilde{\psi}_{i n}-\hat{x} \tilde{\varphi}_{i n} & =\frac{d^{2} \tilde{\psi}_{i n}}{d \hat{x}^{2}},
\end{aligned}
$$


a)

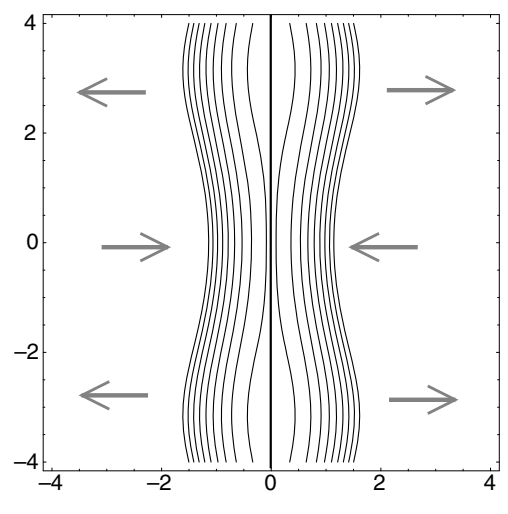

b)

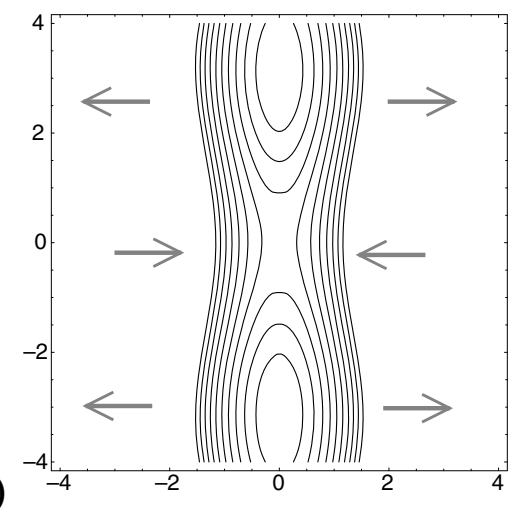

Fig. 2. Equi-contours of the magnetic potential a) Equilibrium (I) and b) Equilibrium (II).

a)

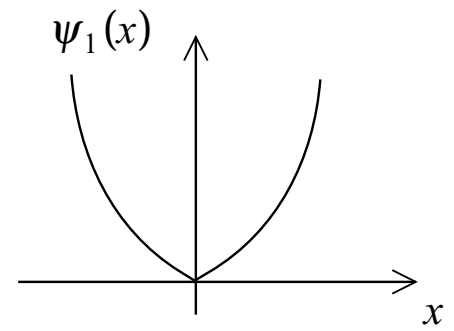

b)

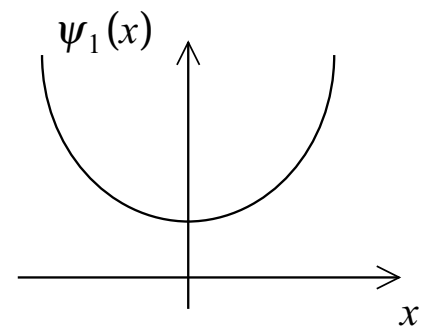

Fig. 3. Perturbed part of the magnetic potential a) Equilibrium (I) and b) Equilibrium (II).

where $\tilde{\psi}_{i n}(\hat{x}, \hat{s})=\tilde{\psi}_{1}(x, s) / \psi_{0}^{\prime \prime}(0) a^{2}, \quad \tilde{\varphi}_{i n}(\hat{x}, \hat{s})=$ $\tilde{\varphi}_{1}(x, s) / v_{A} a, \varphi=\varphi_{1}(x, t) \sin k y$ is the stream function, $\tau_{A}=a / v_{A}, \tau_{R}=4 \pi a^{2} / \eta$ and $v_{A}=\psi_{0}^{\prime \prime}(0) a / \sqrt{4 \pi \rho}$.

The inner-layer solution can be expanded asymptotically (Ara et al., 1978) as

$$
\tilde{\psi}_{i n}(\hat{x}) \approx \psi_{\infty}\left\{1+\frac{\Delta_{i n}^{\prime}}{2} x+\cdots\right\} \quad \hat{x} \rightarrow+\infty,
$$

where

$$
\begin{aligned}
\Delta_{i n}^{\prime}(s) & \equiv \frac{1}{\varepsilon a \psi_{\infty}}\left[\frac{d \tilde{\psi}_{i n}}{d \hat{x}}\right]_{-\infty}^{\infty} \\
& =\frac{-\pi \hat{s}^{5 / 4}}{8 \varepsilon a} \frac{\Gamma\left(\hat{s}^{3 / 2} / 4-1 / 4\right)}{\Gamma\left(\hat{s}^{3 / 2} / 4+5 / 4\right)}, \\
\psi_{\infty} & =\frac{-\tilde{\psi}_{i n}(0, \hat{s})}{\frac{\hat{3}^{3 / 2}}{\hat{s}^{3 / 2}-1} F\left(1,-1 / 2, \hat{s}^{3 / 2} / 4+3 / 4,1 / 2\right)-1},
\end{aligned}
$$

where $F$ is Gauss's Hypergeometric function.

\section{Exact Asymptotic Matching}

Demanding that the solution to the inner-layer equation matches asymptotically with the solution in the outer region yields the exact matching condition (Ishizawa and Tokuda, 2000). On the other hand, in the previous analysis, $\left[d \tilde{\psi}_{i n} / d \hat{x}\right]_{-\infty}^{+\infty}$ is divided by $\tilde{\psi}_{i n}(0, \hat{s})$ instead of $\psi_{\infty}$ in Eq. (12). That is valid only in the constant- $\psi$ approximation realized by neglecting the effect of plasma inertia in the inner layer, because the effect of inertia makes $\tilde{\psi}_{i n}(0, \hat{s})$ deviate from $\psi_{\infty}$. Therefore the matching condition derived by the expansion, Eq. (11), reflects the effect of inertia in the inner layer correctly.

Asymptotic matching of Eqs. (7) and (11) yields the matching conditions as

$$
\begin{aligned}
\tilde{\psi}_{1}(0, s) & =\psi_{0}^{\prime \prime}(0) a^{2} \psi_{\infty}, \\
\Delta_{\text {out }}^{\prime}(s) & =\Delta_{\text {in }}^{\prime}(s) .
\end{aligned}
$$

Combining Eq. (8) and the matching condition, Eq. (15), we have the new Laplace-transformed reconnected flux

$$
\tilde{\psi}_{1}(0, s)=\frac{\Delta_{s}^{\prime} \tilde{\psi}_{e}(s)}{\Delta_{i n}^{\prime}(s)-\Delta_{0}^{\prime}} .
$$

There are two reconnected fluxes to be precise. One is, as introduced above, the reconnected flux at $x=0$, Eq. (16), and represents the temporal change of the quasi-static equilibrium, Eq. (6). The other is a reconnected flux at the origin of the stretched coordinate $\hat{x}=0, \tilde{\psi}_{i n}(0, \hat{s})$, and represents the reconnected flux in the inner layer; it is called the inner-layer reconnected flux in this paper. The inertia of the plasma affects the inner-layer reconnected flux, $\tilde{\psi}_{i n}(0, \hat{s})$, which is then different from the reconnected flux, $\tilde{\psi}_{1}(0, s)$. This difference between the inner-layer reconnected flux and the reconnected flux is given by the matching condition, 
Eq. (14), and Eq. (13) as

$$
\begin{aligned}
\tilde{\psi}_{i n}(0, \hat{s})= & \frac{1}{\psi_{0}^{\prime \prime}(0) a^{2}} \\
& \cdot\left\{1-\frac{\hat{s}^{3 / 2}}{\hat{s}^{3 / 2}-1}\right. \\
& \left.\cdot F\left(1,-1 / 2, \hat{s}^{3 / 2} / 4+3 / 4,1 / 2\right)\right\} \tilde{\psi}_{1}(0, s) .
\end{aligned}
$$

The exact matching gives the Laplace-transformed reconnected fluxes, Eqs. (16) and (17). The inversion of the Laplace transform of Eq. (16) for $|s| \rightarrow \infty$ yields the initial evolution of the reconnected flux (Ishizawa and Tokuda, 2000).

\section{Evolution Equation for Reconnected Flux}

In this section we propose a new method to determine the time evolution of the reconnected flux. Equation (16) can be written as

$$
\tilde{\psi}_{1}(0, s)-\frac{\Delta_{i n}^{\prime}(s)}{\Delta_{0}^{\prime}} \tilde{\psi}_{1}(0, s)=\frac{-\Delta_{s}^{\prime}}{\Delta_{0}^{\prime}} \tilde{\psi}_{e}(s) .
$$

The inversion of the Laplace transform of this equation gives the following inhomogeneous second kind Volterra equation:

$$
\psi_{1}(0, t)+\int_{0}^{t} \psi_{1}(0, \tau) G(t-\tau) d \tau=\frac{-\Delta_{s}^{\prime}}{\Delta_{0}^{\prime}} \psi_{e}(t),
$$

where the kernel $G(t)$ is the Bromwich integral of $\Delta_{i n}^{\prime}(s)$ :

$$
G(t) \equiv \frac{1}{2 \pi i} \int_{\sigma-i \infty}^{\sigma+i \infty} \Delta_{i n}^{\prime}(s) e^{s t} d s
$$

which consists of the sum of residues at the poles and the integral along the branch cut. It is written as

$$
\begin{aligned}
G(t)= & \frac{1}{\tau_{\alpha}}\left\{-\frac{2}{3 \sqrt{\pi}} \exp \left(\frac{t}{\tau_{c}}\right)\right. \\
& -\frac{4}{3 \pi} \sum_{n=1}^{\infty} \frac{\sqrt{n-1 / 4}}{n !} \Gamma(n-1 / 2) \\
& \cdot \exp \left(\frac{-t}{2 \tau_{n}}\right) \sin \left(\frac{\sqrt{3}}{2} \frac{t}{\tau_{n}}\right) \\
& +\frac{1}{3 \pi^{2}} \int_{0}^{\infty} \sqrt{x}|\Gamma(i x-1 / 4)|^{2} \\
& \left.\cdot \exp \left(-(4 x)^{2 / 3} t / \tau_{c}-\pi x\right) d x\right\}
\end{aligned}
$$

where $\tau_{n}=\tau_{c} /(4 n-1)^{2 / 3}, \tau_{\alpha}=-\Delta_{0}^{\prime} \tau_{A} / \pi k$ denote the ideal time scale and $\tau_{c}=\tau_{A}^{2 / 3} \tau_{R}^{1 / 3} /(k a)^{2 / 3}$ is the typical time scale in the inner layer. Note that the amplitude of the tearing mode stability parameter, $\Delta_{0}^{\prime}$, affects to the ideal time scale, $\tau_{\alpha}$. The right-hand side of Eq. (18) represents the externally imposed boundary perturbation.

The time scale in the exponential function in the kernel $G(t)$ is the same as the typical time scale of the inner layer, $\tau_{c} \propto \tau_{A}^{2 / 3} \tau_{R}^{1 / 3}$, because the kernel represents the response of the inner layer to the applied boundary perturbation. At $t=0$ the integral part vanishes and $\psi_{e}(0)=0$, and thus the reconnected flux vanishes at $t=0$ to satisfy the initial condition.

The integral equation for the inner-layer reconnected flux $\psi_{i n}(0, t)$ is deduced in the same way as the above method.

\section{Initial Evolution}

In this section, we preset the initial evolution of the forced reconnection process by use of the integral equation, Eq. (18), reduced in the previous section.

\subsection{Reconnected flux}

Since the integration in Eq. (18) is very small at $t \approx 0$, the reconnected flux increases as $\psi_{1}(0, t) \simeq-\Delta_{s}^{\prime} \psi_{e}(t) / \Delta_{0}^{\prime}=$ $-\Delta_{s}^{\prime} / \Delta_{0}^{\prime}\left\{\psi_{e}^{\prime \prime}(0) t^{2} /\left(2 ! \tau_{e}^{2}\right)+\cdots\right\}$. The initial evolution of the reconnected flux is dominated by the first term of this expansion, therefore its typical time scale is the same as the time scale of the boundary perturbation, $\tau_{e}$, and does not include the Sweet-Parker time scale at all.

We can obtain a higher order expansion (Neumann series) by substituting this into the integration in Eq. (18), and thus, we have

$$
\psi_{1}(0, t)=-\frac{\Delta_{s}^{\prime}}{\Delta_{0}^{\prime}}\left\{\frac{\psi_{e}^{\prime \prime}(0)}{\tau_{e}^{2}} \frac{t^{2}}{2 !}+\frac{\psi_{e}^{\prime \prime}(0)}{\tau_{\alpha} \tau_{e}^{2}} \frac{t^{3}}{3 !}+\cdots\right\} .
$$

Note that the expansion is valid for $\tau_{A} \ll \tau_{\alpha}$ which corresponds to near the ideal marginal stability, $1 \ll\left|\Delta_{0}^{\prime}\right|$, to be precise.

\subsection{Current sheet}

When the boundary perturbation is applied, a current sheet is induced at the resonant surface. The amount of current sheet is represented by the finite jump of the $y$-component of the magnetic field at the resonant surface, $x=0$,

$$
\Delta B_{y}(t) \equiv\left[\frac{\partial \psi_{1}(x, t)}{\partial x}\right]_{-0}^{+0} .
$$

Substituting Eq. (20) into Eq. (21) gives the initial evolution of the current sheet as

$$
\Delta B_{y}(t)=-\Delta_{s}^{\prime}\left\{\frac{\psi_{e}^{\prime \prime}(0)}{3 !} \frac{t^{3}}{\tau_{\alpha} \tau_{e}^{2}}+\frac{\psi_{e}^{\prime \prime}(0)}{\tau_{\alpha}^{2} \tau_{e}^{2}} \frac{t^{4}}{4 !}+\cdots\right\} .
$$

In order to compare Eq. (22) with the result found in previous works, we consider the time evolution of the stability parameter $\Delta^{\prime}(t) \equiv \Delta B_{y} / \psi_{1}(0, t)$. The stability parameter $\Delta^{\prime}(t)$ is negative in the initial evolution and $\Delta^{\prime}(t)=0$ at $t=0$, while it was claimed that $\Delta^{\prime}(t) \rightarrow \infty$ with the positive sign at $t=0$ in previous works. The negative sign of $\Delta^{\prime}(t)$ or $\Delta B_{y}(t)$ implies that the current sheet is induced so as to oppose the progress of the reconnection. However the push caused by the imposed boundary deformation overcomes this resistance to progress the reconnection.

\subsection{Inner-layer reconnected flux}

The reconnected flux derived above is defined at the origin of the outer variable $x=0$. However the limit $x \rightarrow 0$ of the outer variable corresponds to the limit $\hat{x} \rightarrow \infty$ of the inner variable as shown in the asymptotic matching. The magnetic and dynamic structures in the inner layer make the reconnected flux at $x=0$ deviate from the one at $\hat{x}=$ 
0 . Therefore the exact reconnected flux in the inner layer should be defined at $\hat{x}=0$, as shown in Eq. (17). The inner-layer reconnected flux is defined by the inverse of the Laplace transform of $\tilde{\psi}_{i n}(0, s)$. The inverse Laplace transformation of the expansion of Eq. (17) for $|s| \rightarrow \infty$ yields the Taylor-series expansion of the inner-layer reconnected flux:

$$
\psi_{i n}(0, t)=\frac{\Delta_{s}^{\prime}}{\Delta_{0}^{\prime}}\left\{\frac{2 \psi_{e}^{\prime \prime}(0)}{\tau_{e}^{2} \tau_{c}^{3}} \frac{t^{5}}{5 !}+\frac{2 \psi_{e}^{\prime \prime}(0)}{\tau_{\alpha} \tau_{e}^{2} \tau_{c}^{3}} \frac{t^{6}}{6 !}+\cdots\right\} .
$$

Since the initial evolution is dominated by the first term, the time scale of the initial evolution is $\tau_{e}^{2 / 5} \tau_{c}^{3 / 5}$. It is close to the typical time scale of the inner layer, $\tau_{c} \propto \tau_{A}^{2 / 3} \tau_{R}^{1 / 3}$, compared with that of the reconnected flux, $\tau_{e}$. Therefore the time scale of the inner-layer reconnected flux is also significantly different from, and can even be shorter than, the Sweet-Parker time scale. We remark that the expansion is valid for $\tau_{A} \ll \tau_{\alpha}$ which corresponds to near the ideal marginal stability, $1 \ll\left|\Delta_{0}^{\prime}\right|$.

\section{Summary}

We introduced the correct asymptotic matching to improve the boundary layer analysis of forced reconnection due to a boundary perturbation. The correct asymptotic matching yields an integral equation for the time evolution of the reconnected flux. It gives the exact reconnection process which reflects the effect of plasma inertia in the inner layer precisely.

We have shown that the integral equation produces a new time scale at the initial evolution of the forced reconnection that is affected by the time scale of the boundary perturbation. The reconnected flux initially increases on the same time scale as the boundary perturbation, $\tau_{e}$, for near the ideal MHD marginal stability case, $\left|\Delta_{0}^{\prime}\right| \gg 1$. It is different from the Sweet-Parker time scale, $\tau_{s p}=\tau_{A}^{1 / 2} \tau_{R}^{1 / 2}$, for the suddenly imposed boundary perturbation and the modified Sweet-Parker time scale, $\tau_{s p}^{2 /(2+b)} \tau_{e}^{b /(2+b)}$, for the time dependent boundary perturbation, $\psi_{e}(t) \propto\left(t / \tau_{e}\right)^{b}$ in the previous works.

To be precise the reconnection process is described by two reconnected fluxes: the reconnected flux and the inner-layer reconnected flux. The former determines the time evolution of the quasi-static equilibrium of the outer region. The latter is newly introduced and represents the reconnection in the inner layer. The effect of inertia forces these reconnected fluxes to differ. The time scale of the inner-layer reconnected flux is derived from not only the time scale of the boundary perturbation but also the typical time scale of the inner layer, $\tau_{c} \propto \tau_{A}^{2 / 3} \tau_{R}^{1 / 3}$.

The improved theory also yields the new feature of a current sheet induced on the resonant surface. In the initial evolution, the current sheet increases with $\Delta^{\prime}(t)<0$ so as to oppose the progress of the reconnection. In contrast, it was believed to be a typical feature of forced reconnection that $\Delta^{\prime}(t)>0$ and $\Delta^{\prime}(t) \rightarrow+\infty$ at $t=0$, in previous works. The negative sign of the tearing mode stability parameter, $\Delta_{0}^{\prime}<0$, leads to this negative increase in the current sheet, $\Delta^{\prime}(t)<0$. Therefore the negative sign of the current sheet stems from the fact that the original static equilibrium is stable.

Acknowledgments. The authors would like to thank Prof. M. Wakatani at Kyoto University and Dr. M. Azumi and Dr. Y. Kishimoto at JAERI for their useful discussions. One of the authors A. I. is supported by Research Fellowships of the Japan Society for the Promotion of Science for Young Scientists.

\section{References}

Ara, G., B. Basu, B. Coppi, G. Laval, M. N. Rosenbluth, and B. V. Waddell, Magnetic reconnection and $m=1$ oscillations in current carrying plasmas, Ann. Phys., 112, 443-476, 1978.

Fitzpatrick, R. and T. C. Hender, The interaction of resonant magnetic perturbations with rotating plasmas, Phys. Fluids B, 3, 644-673, 1991.

Furth, H. P., J. Killeen, and M. N. Rosenbluth, Finite-resistivity instabilities of a sheet pinch, Phys. Fluids, 6, 459-484, 1963.

Hahm, T. S. and R. M. Kulsrud, Forced magnetic reconnection, Phys. Fluids, 28, 2412-2418, 1985.

Hegna, C. C., J. D. Callen, and R. J. LaHaye, Dynamics of seed magnetic island formation due to geometrically coupled perturbations, Phys. Plasmas, 6, 130-136, 1999.

Ishizawa, A. and S. Tokuda, Improved boundary layer analysis of forced magnetic reconnection due to a boundary perturbation, Phys. Plasmas, 7, 875-882, 2000

Ma, Z. W., X. Wang, and A. Bhattacharjee, Forced magnetic reconnection and the persistence of current sheets in static and rotating plasmas due to a sinusoidal boundary perturbation, Phys. Plasmas, 3, 2427-2433, 1996.

Vekstein, G. E. and R. Jain, Energy release and plasma heating by forced magnetic reconnection, Phys. Plasmas, 5, 1506-1513, 1998.

Vekstein, G. E. and R. Jain, On plasma heating by reconnective magnetic relaxation, Phys. Plasmas, 6, 2897, 1999.

Wang, X. and A. Bhattacharjee, Forced reconnection and current sheet formation in Taylor's model, Phys. Fluids B, 4, 1795-1799, 1992.

Wang, X. and A. Bhattacharjee, Forced reconnection and model locking in rotating cylindrical plasmas, Phys. Plasmas, 4, 748-754, 1997.

Wang, X., A. Bhattacharjee, Z. W. Ma, C. Ren, C. C. Hegna, and J. D. Callen, Structure and dynamics of current sheets at Alfvén resonances in a differentially rotating plasma, Phys. Plasmas, 5, 2291-2296, 1998.

A. Ishizawa (e-mail: ishizawa@center.iae.kyoto-u.ac.jp) and S. Tokuda 